For personal use only. Not to be reproduced without permission of the publisher (editorial@gabi-iournal.net).

\title{
Adjusted indirect comparisons between generics - bioequivalence and interchangeability
}

Professor Laszlo Endrenyi 1, , DSc, PhD; Professor Laszlo Tothfalusi ${ }^{3}, M S c$, PhD

Adjusted indirect comparisons are valuable to establish the bioequivalence and thereby the interchangeability between generic drug products. Limitations should be observed especially when several generics of a drug are marketed.

Keywords: Adjusted indirect comparisons, bioequivalence, generic medicines, interchangeability, multiple generic drug products, switchability

A recent review discussed adjusted indirect comparisons of generic drug products as a tool to establish their bioequivalence and thereby their interchangeability [1]. This is an important issue since often several generic drug products are marketed. Each of them usually will have received regulatory approval on the basis of their demonstrated bioequivalence with the same originator reference drug. This means, directly, that the generics receive marketing authorization, i.e. that they as well as the reference drug may be prescribed and dispensed to patients. Strictly speaking, this replacement applies to individuals who have not received the drug in any of its forms and who are, thus, naïve to it. In this interpretation, regulatory approval of a generic drug product enables its prescribability [2].

It is, however, widely assumed that the approval indicates also that if a patient has been already maintained on one of the approved formulations of a drug then $\mathrm{s} /$ he may be readily switched to another. Consequently, the generic and reference drug products are considered to be switchable within individuals.

It is less clear if switching among various generics formulations may also be undertaken without undue risks. The investigations of indirect comparisons aim to shed light on this question. They explore conditions under which two generic drug products are expected to show bioequivalence when both are bioequivalent to a common reference drug $[1,3]$.
The approach of adjusted indirect comparison is a useful method for assessing the bioequivalence between two generic drug products both of which were shown to be bioequivalent to the same reference drug. The merit of the approach was demonstrated between multiple generic products of several drugs. They included comparisons between, among others, various formulations of cyclosporine, tacrolimus, venlafaxine, olanzapine, atorvastatin, bicalutamide and gabapentin marketed in The Netherlands [4, 5], and tacrolimus formulations in Spain [6]. The method of the adjusted indirect comparison was useful also for the evaluation of bioequivalence between products of first-line antiretroviral drugs, first-line anti-tuberculosis drugs and various antimalarials all of which were in the registry of the World Health Organization (WHO) [3, 7].

Gwaza et al. [3] evaluated six statistical approaches which calculated the width of confidence intervals using either the normal or Student's t-distribution. They found that the most appropriate and simplest indirect contrast of two bioequivalence studies was obtained by their adjusted indirect comparison assuming homogeneous variances [3].

The approach of adjusted comparison should be applied with some care. The conditions of the investigations evaluating the bioequivalence of the two generics with a common reference drug should be comparable. Thus, characteristics of the study groups and the mode of drug administration should be alike. The methodological quality of the investigations should be similar and satisfactory.

The approach has also some constraints and limitations. Computer simulations demonstrated [8] that adjusted indirect comparisons could conclude bioequivalence between generics only when the logarithmically calculated difference between their point estimates was less than $5.5 \%$ in studies having an initially designed power of at least $80 \%$. Furthermore, when the difference was larger, but still did not exceed $14 \%$, then both bioequivalence studies needed to have larger power.

Simulated studies of Karalis et al. [9] also showed that two generics can be switched only when the point estimates of their parameters were similar and the withinsubject variation was small.

The Monte-Carlo simulations investigated the indirect comparison of bioequivalence between two generics. It is expected that when larger numbers of marketed generics are available then increasingly more stringent expectations will be needed for the allowable differences between means and/or the requirements for the designed statistical power. This was the sense of the analysis by Anderson and Hauck [10]. They concluded that with two or three generics, one can be fairly confident of bioequivalence. With five of six generics, this kind of confidence becomes quite low. 
In view of these considerations, Gwaza et al. [8] suggested that regulatory authorities may wish to impose a restraint of a point estimate in the original bioequivalence studies in order to ensure generics interchangeability and to reduce the influence of study design on the outcome.

However, the imposition of such a constraint could have unforeseen consequences. It would amount to the simultaneous imposition of two regulatory requirements. The statistical properties of this regime would certainly be complicated. An example of a similar dual requirement is the expectation, for the determination of bioequivalence between highly-variable drug products, of both a confidence interval criterion and a constraint on the comparative point estimates. Such regulation is applied by both the US Food and Drug Administration (FDA) and the European Medicines Agency (EMA). However, the point estimate and not the confidence interval criterion dominates the regulatory decision when the within-subject variation is very large $[11,12]$. This is contrary to the original intent of the approach. Consequently, caution is warranted for simultaneous applications of two (or more) regulatory criteria.

Current regulations for the demonstration of bioequivalence involve average responses. Often, 90\% confidence intervals around a difference between (frequently logarithmic) average parameters must be within preset regulatory limits. This also means that, in principle and in the extreme case, $10 \%$ of randomly obtained average differences could be outside the regulatory limits. The percentage potentially falling outside the limits could be much larger if the difference between each possible difference of individual parameters is considered.

These thoughts have little importance when the $90 \%$ confidence interval is well within the regulatory limits, i.e. when the margin is wide for reaching a decision on the prevalence of bioequivalence. This is the case in the vast majority of comparisons. However, the above scenario could be of concern under some conditions. For instance, caution should be exercised for the indirect comparisons of generics. For example, some investigations found that most deviations between marketed generic drug products satisfied the usual 80-125\% criterion but by no means all of them [4, 8]. In view of the arguments above, this concern is enhanced when an increasing number of generics is on the market.

In summary, the approach of indirect comparisons is a valuable tool for the assessment of bioequivalence between generic drug products each of which is bioequivalent with the same reference drug. Important information has already been revealed about the bioequivalence between generics formulations of a wide range of drugs. This has led to conclusions on the interchangeabilty of these generic drug products and the belief that generics formulations are generally switchable. However, some caution should be exercised in this regard especially when the number of marketed generics is large. The suggestion by the authors of the recent review on the topic [1] is well advised: in order to ensure the interchangeability among generics, the ratios of the point estimates between the generics should be small and the original studies should be sufficiently powered.

\section{Competing interests: None.}

Provenance and peer review: Commissioned, internally peer reviewed.

\section{Co-author}

Professor Laszlo Tothfalusi, MSc, PhD Semmelweis University, Department of Pharmacodynamics, HU-1445 Budapest, Hungary

\section{References}

1. Gwaza L, Gordon J, Potthast H, Maliepaard M, Welink J, Leufkens $\mathrm{H}$, et al. Assessment of the interchangeability between generics. Generics and Biosimilars Initiative Journal (GaBI Journal). 2016; 5(2):55-9. doi:10.5639/gabij.2016.0502.015

2. Anderson S, Hauck WW. Consideration of individual bioequivalence. J Pharmacokinet Biopharm. 1990;18(3):259-73.

3. Gwaza L, GordonJ, WelinkJ, Potthast H, Hansson H, Stahl MM, et al. Statistical approaches to indirectly compare bioequivalence between generics: a comparison of methodologies employing artemether/lumefantrine $20 / 120 \mathrm{mg}$ tablets as prequalified by WHO. Eur J Clin Pharmacol 2012;68(12):1611-8.

4. Yu Y, Teerenstra S, Neef C, Burger D, Maliepaard M. Investigation into the interchangeability of generic formulations using immunosuppressants and a broad selection of medicines. Eur J Clin Pharmacol. 2015;71(8):979-90.

5. Yu Y, Teerenstra S, Vanmolkot F, Neef C, Burger D, Maliepaard M. Interchangeability of gabapentin generic formulations in the Netherlands: a comparative bioavailability study. Clin Pharmacol Ther. 2013;94(4):519-24.

6. Herranz M, Morales-Alcelay S, Corredera-Hernández MT, de la Torre-Alvarado JM, Blázquez-Pérez A, Suárez-Gea ML, et al. Bioequivalence between generic tacrolimus products marketed in Spain by adjusted indirect comparison. Eur J Clin Pharmacol. 2013;69(5):1157-62.

7. Gwaza L, Gordon J, Welink J, Potthast H, Leufkens H, Stahl M, et al. Adjusted indirect treatment comparison of the bioavailability of WHO-prequalified first-line generic antituberculosis medicines. Clin Pharmacol Ther. 2014;96(5):580-8.

8. Gwaza L, Gordon J, Potthast H, Welink J, Leufkens H, Stahl M, et al. Influence of point estimates and study power of bioequivalence studies on establishing bioequivalence between generics by adjusted indirect comparisons. Eur J Clin Pharmacol. 2015;71(9):1083-9.

9. Karalis V, Macheras P, Bialer M. Generic products of antiepileptic drugs: a perspective on bioequivalence, bioavailability, and formulation switches using Monte Carlo simulations. CNS Drugs. 2014;28(1):69-77.

10. Anderson S, Hauck WW. The transitivity of bioequivalence testing: potential for drift. Int J Clin Pharmacol Ther. 1996;34(9):369-74.

11. Endrenyi L, Tothfalusi L. Regulatory and study conditions for the determination of bioequivalence of highly variable drugs. J Pharm Pharm Sci. 2009;12(1):138-49.

12. Haidar SH, Makhlouf F, Schuirmann DJ, Hyslop T, Davit B, Conner D, et al. Evaluation of a scaling approach for the bioequivalence of highly variable drugs. AAPS J. 2008;10(3):450-4

DOI:10.5639/gabij.2016.0502.014

Copyright $\odot 2016$ Pro Pharma Communications International 